\title{
Amyloid- $\beta$ Imaging with Pittsburgh Compound B and Florbetapir: Comparing Radiotracers and Quantification Methods
}

\author{
Susan M. Landau ${ }^{1-3}$, Christopher Breault ${ }^{3}$, Abhinay D. Joshi ${ }^{3}$, Michael Pontecorvo ${ }^{3}$, Chester A. Mathis ${ }^{4}$, \\ William J. Jagust ${ }^{1,2,5}$, and Mark A. Mintun ${ }^{3}$, for the Alzheimer's Disease Neuroimaging Initiative \\ ${ }^{1}$ Helen Wills Neuroscience Institute, University of California, Berkeley, California; ${ }^{2}$ Life Sciences Division, Lawrence Berkeley \\ National Laboratory, Berkeley, California; ${ }^{3}$ Avid Radiopharmaceuticals, Inc., Philadelphia, Pennsylvania; ${ }^{4}$ Department of Radiology, \\ PET Facility, University of Pittsburgh, Pittsburgh, Pennsylvania; and ${ }^{5}$ School of Public Health, University of California, Berkeley, \\ California
}

\begin{abstract}
${ }^{11} \mathrm{C}$-Pittsburgh compound $\mathrm{B}\left({ }^{11} \mathrm{C}-\mathrm{PiB}\right)$ and ${ }^{18} \mathrm{~F}$-florbetapir amyloid- $\beta(A \beta)$ PET radioligands have had a substantial impact on Alzheimer disease research. Although there is evidence that both radioligands bind to fibrillar $A \beta$ in the brain, direct comparisons in the same individuals have not been reported. Here, we evaluated $\mathrm{PiB}$ and florbetapir in a retrospective convenience sample of cognitively normal older controls, patients with mild cognitive impairment, and patients with Alzheimer disease from the Alzheimer's Disease Neuroimaging Initiative (ADNI). Methods: From the ADNI database, 32 participants were identified who had undergone at least 1 PiB study and subsequently underwent a florbetapir study approximately $1.5 \mathrm{y}$ after the last $\mathrm{PiB}$ study. Cortical $\mathrm{PiB}$ and florbetapir retention was quantified using several different methods to determine the effect of preprocessing factors (such as smoothing and reference region selection) and image processing pipelines. Results: There was a strong association between $\mathrm{PiB}$ and florbetapir cortical retention ratios (Spearman $\rho=0.86-0.95$ ), and these were slightly lower than cortical retention ratios for consecutive $\mathrm{PiB}$ scans (Spearman $\rho=0.96-0.98$ ) made approximately 1.1 y apart. Cortical retention ratios for $A \beta$-positive subjects tended to be higher for $\mathrm{PiB}$ than for florbetapir images, yielding slopes for linear regression of florbetapir against PiB of 0.59-0.64. Associations between consecutive PiB scans and between PiB and florbetapir scans remained strong, regardless of processing methods such as smoothing, spatial normalization to a PET template, and use of reference regions. The PiB-florbetapir association was used to interconvert cutoffs for $A \beta$ positivity and negativity between the 2 radioligands, and these cutoffs were highly consistent in their assignment of $A \beta$ status. Conclusion: $\mathrm{PiB}$ and florbetapir retention ratios were strongly associated in the same individuals, and this relationship was consistent across several data analysis methods, despite scan-rescan intervals of more than a year. Cutoff thresholds for determining positive or negative $A \beta$ status can be reliably transformed
\end{abstract}

Received Jun. 4, 2012; revision accepted Aug. 3, 2012.

For correspondence or reprints contact: Susan M. Landau, 118 Barker Hall, MC \#3190, University of California, Berkeley, CA 94720-3190.

E-mail: slandau@berkeley.edu

Published online Nov. 19, 2012

COPYRIGHT (C 2013 by the Society of Nuclear Medicine and Molecular Imaging, Inc. from $\mathrm{PiB}$ to florbetapir units or vice versa using a population scanned with both radioligands.

Key Words: amyloid- $\beta$; Alzheimer's disease; PET imaging

J Nucl Med 2013; 54:70-77

DOI: 10.2967/jnumed.112.109009

$\mathbf{P}$ ET of fibrillar amyloid- $\beta$ (A $\beta)$ in vivo has had a substantial impact on Alzheimer disease (AD) research. The first selective radioligand developed for this purpose, ${ }^{11} \mathrm{C}$ Pittsburgh compound $\mathrm{B}(\mathrm{PiB})$, has played a critical role in this research, contributing to the increasing understanding of disease development and the presence of cortical $A \beta$ in the cognitively normal older population. Because the 20-min half-life of ${ }^{11} \mathrm{C}$-labeled radioligands such as $\mathrm{PiB}$ restricts their use to centers with a cyclotron on-site, the recent development of ${ }^{18} \mathrm{~F}$-labeled radioligands such as florbetapir promises to facilitate the accessibility of $A \beta$ imaging in $A D$ research. The longer half-life of ${ }^{18} \mathrm{~F}$ (110 min) makes it possible to transport these radioligands from production site to PET scanner.

Validation studies have established that cortical $A \beta$ measured with florbetapir can be detected in cognitively normal subjects, in patients with mild cognitive impairment (MCI), and in patients with Alzheimer disease $(\mathrm{AD})(1,2)$ in proportions comparable to those reported for PiB (3). Furthermore, autopsy studies in which individuals were scanned with $\mathrm{PiB}(4)$ and florbetapir $(5,6)$ before death have provided additional evidence that both PET ligands bind to fibrillar amyloid in cortex. However, data directly comparing PiB and florbetapir in the same individuals are still limited (7).

The goal of this study was to compare measurements of cortical retention ratios of $\mathrm{PiB}$ and florbetapir in a subset of Alzheimer's Disease Neuroimaging Initiative (ADNI) participants who underwent 2 consecutive PiB imaging sessions followed by 1 florbetapir scanning session at approximately 1 - to $2-y$ intervals. Because the precise 
quantification of cortical $A \beta$ may be influenced by various image processing and analysis methods, we examined the influence of several of these factors. Finally, we examined the feasibility of transforming cutoff thresholds for $A \beta$ positivity, with the goal of working toward standardization in $\mathrm{A} \beta$ imaging quantification.

\section{MATERIALS AND METHODS \\ ADNI}

Our study population was drawn from the ADNI, a longitudinal multisite study supported by the National Institutes of Health, private pharmaceutical companies, and nonprofit organizations, with approximately 50 medical center and university sites across the United States and Canada (www.loni.ucla.edu/ADNI). The first ADNI protocol, ADNI1, enrolled approximately 800 participants (volunteers with normal cognition, a group initially recruited as $\mathrm{MCI}$ and now termed late MCI, and AD patients) with multiple longitudinal biomarker and cognitive measurements at 6- to 12-mo intervals for 2-4 y. A subset of 102 ADNI1 subjects underwent up to $4 \mathrm{PiB}$ scanning sessions at approximately yearly intervals, and a further subset of these individuals subsequently underwent florbetapir scanning, which started in May 2010 with a new phase of the study, ADNI GO, and has continued during ADNI2, which is ongoing.

\section{Participants}

We studied 32 ADNI participants (8 enrolled as cognitively normal and 24 diagnosed as MCI at enrollment) who as of February 2012 had completed at least $1 \mathrm{PiB}$ scanning session and 1 florbetapir session at approximately 1 - to 2 -y intervals. All participants gave written informed consent that was approved by the Internal Review Board of each participating institution. Thirty-one of 32 participants underwent at least $2 \mathrm{PiB}$ sessions.

Participants underwent apolipoprotein E genotyping and were monitored longitudinally at 6- to 12 -mo intervals for cognitive function (e.g., mini-mental state examination, Alzheimer's Disease Assessment Scale-cognitive subscale) and possible changes in diagnosis. A total of 13 of 32 subjects had a change in diagnosis between ADNI1 enrollment and their florbetapir scan. Of the 8 cognitively normal subjects, 3 converted to MCI between enrollment and their florbetapir imaging session. Of the $24 \mathrm{MCI}$ subjects, 1 reverted to cognitively normal and 9 converted to $\mathrm{AD}$ ( 5 of these conversions occurred between the second $\mathrm{PiB}$ [PiB2] and florbetapir scanning sessions).

\section{PET and Processing}

$\mathrm{PiB}$ and florbetapir image data were acquired from a variety of PET scanners and sites nationwide. Image data are available at 3 levels of preprocessing (raw, unsmoothed, and smoothed) as described online (8). Briefly, the 3 preprocessing phases involve cumulative levels of processing as follows. First, the raw dataset was acquired in four 5-min frames 50-70 min after injection. The 4 frames were coregistered, but data were not at a uniform resolution or smoothing kernel. Second, for the unsmoothed dataset, the raw dataset was averaged and interpolated to a uniform image and voxel size $\left(160 \times 106 \times 96,1.5 \mathrm{~mm}^{3}\right)$. Third, for the smoothed dataset, the unsmoothed dataset was smoothed to a uniform resolution $(8 \mathrm{~mm}$ in full width at half maximum) to account for differences between scanners (9).

\section{Data Analysis}

$\mathrm{PiB}$ and florbetapir image data were analyzed using 2 processing streams. The PET-template analysis method was described in a separate study (10). This method was applied to the raw and unsmoothed datasets. Briefly, image data were spatially normalized to standard atlas coordinates in Talairach space using statistical parametric mapping software (11). Mean tracer retention was calculated for 6 predefined target cortical regions of interest (medial orbital frontal, temporal, parietal, anterior cingulate, posterior cingulate, and precuneus) that resulted from a statistical contrast of AD patients and cognitively normal subjects $(1)$.

The Freesurfer method for quantifying cortical $A \beta$ was applied to the unsmoothed and smoothed datasets. This method was described in detail elsewhere $(2,12)$ and online (13). Structural 1.5-T or 3-T MRI scans (T1-weighted images) were used to define cortical regions of interest and the cerebellar reference region. In general, 2 structural MRI scans were acquired at each visit across several years of follow-up, with the result that several MR images were available for each subject. For processing the PiB images, we chose the T1 scans acquired concurrently with (or closest in time to) the first $\mathrm{PiB}$ scan; and for the florbetapir processing, we chose the T1 scans acquired concurrently with (or closest in time to) the florbetapir scan. Structural MR images were segmented and parceled into individual cortical regions with Freesurfer (version 4.5.0; surfer. nmr.mgh.harvard.edu/) and subsequently used to extract mean $\mathrm{PiB}$ and florbetapir cortical retention ratios from gray matter within lateral and medial frontal, anterior and posterior cingulate, lateral parietal, and lateral temporal regions.

To examine several reference regions, the unscaled cortical means for each analysis method were divided by mean retention in the following 3 reference regions: brain stem-pons, whole cerebellum (white and gray matter), and cerebellar gray matter, yielding 3 cortical retention ratios for each preprocessing method. Because Freesurfer creates a brain stem, but not pons, region as part of its automated processing stream, the brain stem was used for the Freesurfer processing analysis method and the pons was used for the PET-template processing method.

To summarize, for each of 3 PET sessions (2 PiB scans and 1 florbetapir scan), every subject had cortical retention ratios for 2 levels of processing and 2 analysis methods (raw and unsmoothed for the PET-template method and unsmoothed and smoothed for the Freesurfer method), using 3 reference regions (brain stem-pons, whole cerebellum, cerebellar gray matter), resulting in 36 mean cortical retention ratios per subject that were compared in subsequent statistical analyses.

\section{Statistical Methods}

Differences between diagnostic groups were assessed with independent-samples $t$ tests and $\chi^{2}$ tests. Correlations between cortical PiB and florbetapir measurements using different levels of processing, analysis methods, and reference regions were assessed using Spearman rank coefficients $(\rho)$ to account for the nonnormally distributed nature of the cortical PET means.

Regression equations ( $y=$ slope $x x+$ intercept) representing individual cortical measurements obtained during different scanning sessions (first scan [PiB1] vs. PiB2, PiB2 vs. florbetapir) were used to convert cutoff thresholds between tracers and processing methods.

\section{RESULTS}

A summary of and demographic information for participants are shown in Table 1. The diagnostic groups did not differ in age at scanning sessions, education, apolipoprotein E4 status, or sex. Participants diagnosed as MCI at enrollment had lower mini-mental state examination $(P=0.01)$ 
TABLE 1

Demographic and Descriptive Information for Study Population

\begin{tabular}{|c|c|c|c|}
\hline \multirow[b]{2}{*}{ Parameter } & \multirow[b]{2}{*}{$\begin{array}{l}\text { Total sample } \\
\quad(n=32)\end{array}$} & \multicolumn{2}{|c|}{ Diagnosis at enrollment } \\
\hline & & $\begin{array}{l}\text { Normal cognition } \\
\quad(n=8)\end{array}$ & $\begin{array}{c}\mathrm{MCl} \\
(n=24)\end{array}$ \\
\hline Duration of follow-up (y) & 4.3 & 4.3 & 4.3 \\
\hline Mean age $( \pm S D)$ at $\mathrm{PiB} 2 \mathrm{scan}(\mathrm{y})$ & $75.7 \pm 6.6$ & $77.6 \pm 3.9$ & $75.1 \pm 7.3$ \\
\hline Mean age $( \pm S D)$ at florbetapir scan $(y)$ & $77.3 \pm 6.5$ & $79.3 \pm 3.7$ & $76.7 \pm 7.2$ \\
\hline $\begin{array}{l}\text { Mean time }( \pm \mathrm{SD}) \text { between PiB1 and } \\
\text { PiB2 scans }(\mathrm{y})\end{array}$ & $1.1 \pm 0.3$ & $1.0 \pm 0.08$ & $1.1 \pm 0.3$ \\
\hline $\begin{array}{l}\text { Mean time ( } \pm \text { SD) between PiB2 and florbetapir } \\
\text { scans (y) }\end{array}$ & $1.5 \pm 0.7$ & $1.6 \pm 0.8$ & $1.4 \pm 0.6$ \\
\hline Sex, female (\%) & 31 & 25 & 33 \\
\hline Mean number of years $( \pm S D)$ of education & $16.1 \pm 3.0$ & $15.9 \pm 3.0$ & $16.1 \pm 3.0$ \\
\hline Apolipoprotein E4 carriers (\%) & 53 & 50 & 54 \\
\hline Mean MMSE score $( \pm S D)$ at florbetapir scan & $25.2 \pm 6.0$ & $28.3 \pm 1.7$ & $24.1 \pm(.6$ \\
\hline Mean ADAS-cog score ( \pm SD) at florbetapir scan & $13.2 \pm 12.3$ & $6.8 \pm 4.7$ & $15.4 \pm 13.3$ \\
\hline Mean PiB2 cortical retention & $1.51(95 \% \mathrm{Cl}, 1.31-1.71)$ & $1.34(95 \% \mathrm{Cl}, 0.99-1.68)$ & $1.57(95 \% \mathrm{Cl}, 1.31-1.82)$ \\
\hline Mean florbetapir cortical retention & $1.25(95 \% \mathrm{Cl}, 1.13-1.38)$ & $1.13(95 \% \mathrm{Cl}, 0.96-1.30)$ & $1.29(95 \% \mathrm{Cl}, 1.13-1.45)$ \\
\hline \multicolumn{4}{|l|}{ Diagnosis at PiB2 scan $(n)$} \\
\hline Normal cognition & 6 & 5 & 1 \\
\hline $\mathrm{MCl}$ & 22 & 3 & 19 \\
\hline$A D$ & 4 & 0 & 4 \\
\hline \multicolumn{4}{|l|}{ Diagnosis at florbetapir scan $(n)$} \\
\hline Normal cognition & 6 & 5 & 1 \\
\hline $\mathrm{MCl}$ & 17 & 3 & 14 \\
\hline$A D$ & 9 & 0 & 9 \\
\hline
\end{tabular}

95\% confidence intervals (95\% Cls) are for raw data processed using PET-template method. Subject diagnostic groups are based on diagnosis at enrollment, but changes in diagnosis between enrollment, PiB2, and florbetapir scanning sessions are noted. Summary cognitive scores (mini-mental state examination [MMSE], Alzheimer's Disease Assessment Scale-cognitive subscale [ADAS-cog]) are given for participants' most recent imaging session.

and higher Alzheimer's Disease Assessment Scale-cognitive subscale scores $(P=0.01)$ than did participants diagnosed as cognitively normal at enrollment.

There was an average of $2.5 \pm 0.6 \mathrm{y}$ total between the PiB1 and florbetapir scans. The interval between the PiB1 and PiB2 scans $(1.1 \pm 0.3 \mathrm{y})$ was shorter by about 4 mo on average than the interval between the $\mathrm{PiB} 2$ and florbetapir scans $(P=0.04 ; \mathrm{PiB} 2$ to florbetapir, $1.5 \pm 0.7 \mathrm{y})$.

Figure 1 shows $\mathrm{PiB}$ and florbetapir scans for a representative cognitively normal control (who remained normal throughout all scanning sessions) with low tracer retention throughout the cortex ( $\mathrm{PiB}$ cortical retention ratio, 0.95; florbetapir cortical retention ratio, 0.94) and an MCI participant who converted to $\mathrm{AD}$ before the first $\mathrm{PiB}$ scanning session and who showed high retention of both tracers in the cortex relative to the cerebellum ( $\mathrm{PiB}$ cortical retention ratio, 1.98; florbetapir cortical retention ratio, 1.37). Tracer retention throughout the white matter was visible for both subjects.

\section{Effect of Reference Region}

The effect of reference region on the association between the second $\mathrm{PiB}$ and florbetapir scans is shown in Figure 2, using raw data processed with the PET-template method. The slopes and the correlation coefficients showed minimal change across normalization regions (pons, Spearman $\rho=$ 0.94; whole cerebellum, Spearman $\rho=0.95$; and cerebellar gray matter, Spearman $\rho=0.92$ ). However, the range of cortical retention values differed across normalization regions such that cortical retention ratios were greatest for $\mathrm{PiB}$ and florbetapir cortical means normalized by cerebellar gray matter, moderate for data normalized by the whole cerebellum, and smallest for data normalized by the pons. Additionally, individuals whose diagnosis changed during the follow-up period showed an association between PiB2-florbetapir values similar to that of individuals who maintained a stable diagnosis, despite the 1.5-y (on average) interval between the PiB and florbetapir scans.

Therefore, we did not label subjects separately on the basis of diagnosis in subsequent analyses. We also focused primarily on cortical retention ratios normalized by the whole cerebellum in subsequent analyses.

\section{Effect of Image Preprocessing and Analysis}

The effect of data preprocessing on the relationship between consecutive $\mathrm{PiB}$ scans or between the PiB2 and florbetapir scans was minimal overall (Fig. 3). All data shown in Figure 3 are ratios of mean cortical retention to whole cerebellum retention, but associations were comparable using the other reference regions. For the association between PiB1 and PiB2 scans (Fig. 3, left), slopes ranged from 0.97 to 1.0, and correlation coefficients (Spearman $\rho$ ) ranged from 0.96 to 0.98 regardless of the level of data processing (raw [Fig. 3A], 


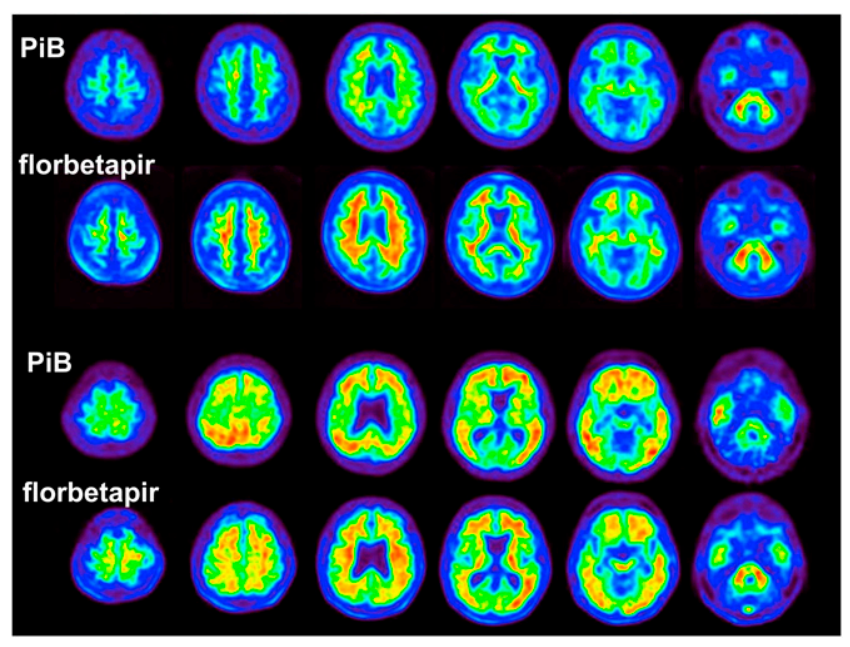

FIGURE 1. Axial slices of $\mathrm{PiB}$ and florbetapir scans are shown for 2 representative subjects, cognitively normal control with low tracer retention (top) and $A D$ patient with high tracer retention in cortex relative to cerebellum, reflecting widespread fibrillar amyloid (bottom).

unsmoothed [Figs. 3B and 3C], smoothed [Fig. 3D]). The PET-template (Fig. 3B) and Freesurfer (Fig. 3C) analysis methods resulted in nearly identical slopes and correlations.

The association between PiB2 and florbetapir had lower slopes (range, 0.59-0.64) and slightly lower correlation coefficients ( $\rho$ range, 0.86-0.95) than did the PiB1-PiB2 associations. Lower slopes for the PiB2-versus-florbetapir association resulted from cortical retention values that were higher for PiB than florbetapir in subjects in the higher range for both tracers. These higher values resulted in cortical retention ratios with a narrower range for florbetapir than for PiB. For example, for raw data processed with the PETtemplate method, $\mathrm{PiB}$ values ranged from 0.86 to 2.67 (a difference of 1.8, dynamic range [ratio] of 3.1) whereas florbetapir values ranged from 0.84 to 2.26 (a difference of 1.4, dynamic range [ratio] of 2.7) (Fig. 3A, right).
The level of preprocessing minimally affected these measurements, such that the highest correlation was observed with raw data $(\rho=0.95)$ and the lowest correlation was observed with the smoothed dataset $(\rho=0.86)$. To further investigate the effect of different levels of preprocessing (raw, unsmoothed, and smoothed) and data analysis methods (PET template and Freesurfer), we converted a preselected PiB cortical-to-gray cerebellum threshold value of 1.47 to corresponding florbetapir values using the 4 regression equations (Fig. 3, right) representing PiB values plotted against florbetapir. The resulting florbetapir values ranged from 1.21 to 1.25 , indicating that the effect of image preprocessing and analysis accounted for about $3 \%$ variability in florbetapir values.

\section{Percentage Change Between Scans}

We used the raw data processed with the PET-template method to calculate the average numeric difference and average percentage change in cortical retention ratios measured for the $\mathrm{PiB} 1$ versus $\mathrm{PiB} 2$ and $\mathrm{PiB} 2$ versus florbetapir scans (Table 2). Difference and percentage change values were reasonably consistent with each other and differed slightly depending on which reference region was used. Collapsing across reference regions, $\mathrm{PiB}$ cortical retention ratios increased by an average of $2.7 \%$ (average of $5.3 \%$ using the absolute values of percentage change) between the 2 consecutive $\mathrm{PiB}$ sessions, whereas $\mathrm{PiB}$ cortical retention ratios were $13.3 \%$ higher (16.3\% absolute percentage change) than those of florbetapir. Higher percentage change values were driven by subjects with greater uptake, indicating that $\mathrm{PiB}$ cortical retention ratios were disproportionately higher than florbetapir values for subjects as amyloid deposition increased.

\section{Thresholds for A $\beta$-Positive and -Negative Status}

We compared 2 recently published cutoff thresholds for establishing $A \beta$ positivity and negativity $(10,14)$. A receiveroperating-characteristic analysis of 17 ADNI cognitively normal and $15 \mathrm{AD}$ subjects with smoothed $\mathrm{PiB}$ scans

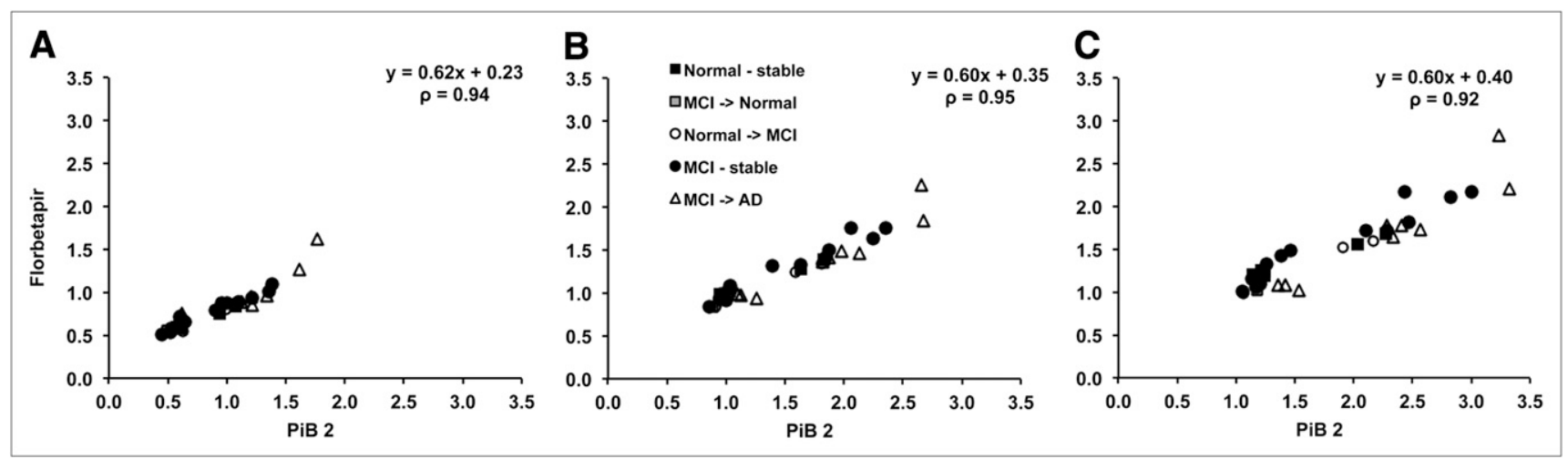

FIGURE 2. Cortical retention ratios are shown for consecutive PiB and florbetapir scans obtained for same participants and processed using pons (A), whole cerebellum (B), and cerebellar gray matter (C) for intensity normalization. Initial diagnosis at enrollment and any subsequent diagnostic change are represented by shape markers. Stable normal cognition or $\mathrm{MCl}$ diagnosis is represented with solid shapes, individuals who progressed are represented with unfilled shapes, and single individual who regressed from $\mathrm{MCl}$ to normal is represented with gray-filled square. Raw data were analyzed with PET-template method. Regression equations and Spearman rank correlation coefficients ( $\rho$ ) are shown for each scatterplot. 


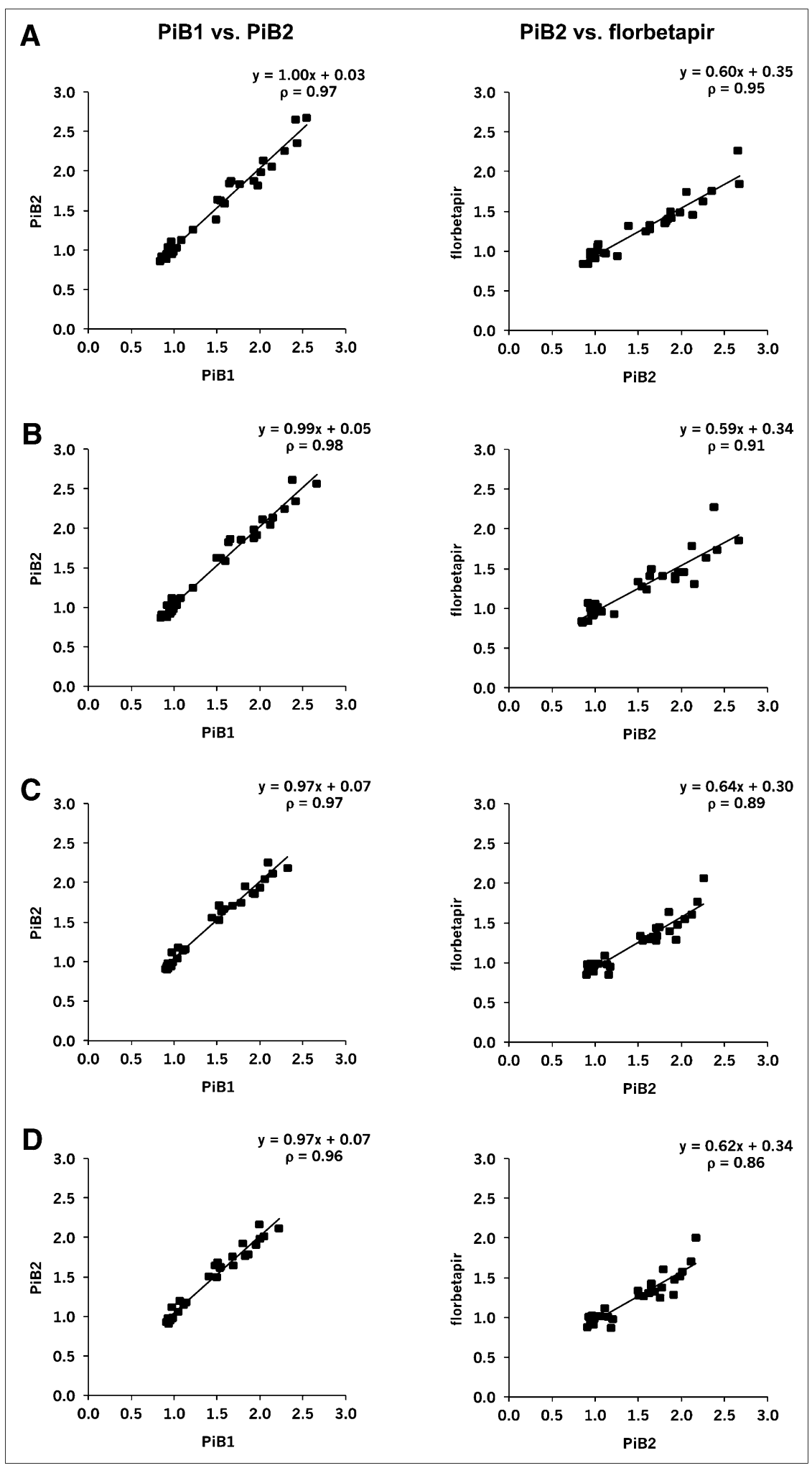

FIGURE 3. Cortical retention ratios for 2 consecutive $\mathrm{PiB}$ scans and $\mathrm{PiB}$ scan followed by florbetapir scan obtained for same participants are compared at different levels of preprocessing and data analysis methods. All cortical retention ratios were normalized using whole cerebellum. Top 2 rows show raw data (not at uniform voxel size or smoothing) (A) and unsmoothed data (at uniform voxel size but not uniform smoothing) (B), both processed using PETtemplate method. Bottom 2 rows show unsmoothed (C) and smoothed data (uniform voxel size and smoothing) (D) processed with Freesurfer method. Thus, middle 2 rows both show unsmoothed data that differs only on basis of which processing method was used (PET template [B], Freesurfer [C]). Regression equations and Spearman rank correlation coefficients $(\rho)$ are shown for each scatterplot.

downloaded from LONI, processed using the Freesurfer method as described here, and intensity-normalized by cerebellar gray matter resulted in an optimal PiB cutoff of 1.47 for isolating $\mathrm{AD}$ patients from controls $(12,14)$. A separate florbetapir study that included a sample of 21 young, healthy subjects determined that a cutoff of 1.10 was the upper $95 \%$ confidence interval above the cortical mean for the young controls. Florbetapir scans were processed using the PETtemplate method as described here and normalized by the whole cerebellum (10). This cutoff was further supported by 
TABLE 2

Mean Difference and Percentage Change Between Consecutive Scans Across All Participants

\begin{tabular}{|c|c|c|c|c|c|c|}
\hline \multirow[b]{2}{*}{ Parameter } & \multicolumn{3}{|c|}{ PiB1 vs. PiB2 } & \multicolumn{3}{|c|}{ PiB2 vs. florbetapir } \\
\hline & Pons & Whole cerebellum & Cerebellar gray & Pons & Whole cerebellum & Cerebellar gray \\
\hline Difference & $0.05(0.20)$ & $0.10(0.33)$ & $0.13(0.40)$ & $-0.12(0.16)$ & $-0.28(0.24)$ & $-0.37(0.32)$ \\
\hline Percentage change & $1(6)$ & $3(6)$ & $4(7)$ & $-8(15)$ & $-15(11)$ & $-17(13)$ \\
\hline
\end{tabular}

Data in parentheses are SDs, reflecting increases in tracer retention between first and second PiB scans and between second PiB and florbetapir scans, separately for each reference region. Positive value represents mean increase from PiB1 to PiB2 or from PiB2 to florbetapir, whereas negative value represents decrease.

another study showing that no individuals with a cortical florbetapir mean (calculated using the same method as was used in the study of Joshi et al. (10)) less than approximately 1.10 had an intermediate to high likelihood of AD, according to the criteria of the National Institute on Aging/Reagan Institute based on neuropathology at autopsy (5).

Because the PiB cutoff of 1.47 and the florbetapir cutoff of 1.10 were obtained using different processing methods, we compared the cutoffs by transforming PiB-cerebellar gray matter normalization units to florbetapir-whole cerebellum normalization and vice versa. We plotted florbetapir means, based on the raw dataset and normalized by the whole cerebellum, as in the study of Joshi et al. (10) against $\mathrm{PiB}$ data that were smoothed and normalized by cerebellar gray matter, as in the study of Jagust et al. (Fig. 4) (14). We used the resulting regression equation $(y=0.67 x+0.15)$ to convert the $\mathrm{PiB}$ cutoff of 1.47 into 1.13 florbetapir units. Similarly, plotting $\mathrm{PiB}$ means against florbetapir means resulted in a regression equation $(y=1.23 x+0.09)$ that we used to convert the florbetapir cutoff of 1.10 to 1.44 in

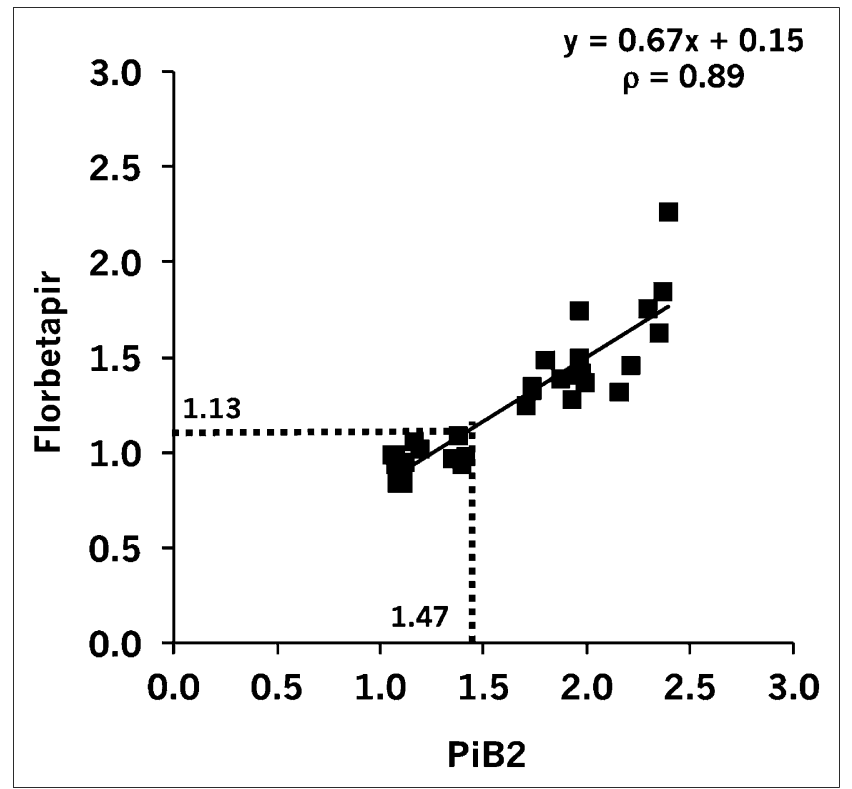

FIGURE 4. PiB threshold of 1.47 (14) that is based on data normalized to cerebellar gray matter can be converted to florbetapir threshold of 1.13, using raw data and whole-cerebellum normalization.
PiB units. The use of different levels of image preprocessing changed the resulting cutoff values by $3 \%$ or less.

When the florbetapir cutoff of 1.10 was applied to the population, 18 of 32 participants were categorized as positive based on florbetapir scans ( $56 \%$ total; $2 / 6$ cognitively normal controls, 10/17 MCI patients, and 6/9 AD patients, based on diagnosis at the time of florbetapir). When the PiB cutoff of 1.47 was applied to $\mathrm{PiB} 2$ scans, the positive-negative categorization was consistent for 31 of 32 subjects (the discrepant subject was a stable MCI individual), indicating 97\% agreement between the 2 thresholds in this population. Finally, the positive-negative categorizations for the 2 florbetapir cutoffs (the 1.10 cutoff from the study of Joshi et al. (10) or the 1.13 PiB-derived cutoff from the study of Jagust et al. (14)) were consistent in $100 \%$ of subjects.

\section{DISCUSSION}

${ }^{11} \mathrm{C}-\mathrm{PiB}$ and ${ }^{18} \mathrm{~F}$-florbetapir cortical retention ratios were highly correlated in a subset of the ADNI population $(n=$ 32). Correlations between PiB2 and florbetapir cortical retention ratios were strong, regardless of which of the processing methods were used (Spearman $\rho=0.86-0.95$ ), with the highest association resulting from image data that had no adjustments for uniform voxel size and smoothing. In addition, the range of florbetapir values was small relative to $\mathrm{PiB}$, and this difference in radioligand scales could be accounted for by converting an existing PiB cutoff to florbetapir units (and vice versa). The resulting cutoffs were highly consistent in their assignment of $\mathrm{A} \beta$-positive and -negative status for all subjects and closely matched previously reported, independently derived cutoffs for $\mathrm{PiB}$ and florbetapir.

The reduced range of florbetapir retention values, compared with $\mathrm{PiB}$, has been reported previously (7) and for other ${ }^{18} \mathrm{~F}$ tracers such as florbetaben (15). This phenomenon may be related to the influence of nonspecific white matter retention on cortical and reference regions (via partial-volume effects) that has been observed with florbetapir previously (16) and with other ${ }^{18} \mathrm{~F}$ ligands, including flutemetamol (17), florbetaben $(15,18)$, and AZD-4694 (19). However, nonspecific retention in white matter has been reported with $\mathrm{PiB}$ as well (20). Because we found that $\mathrm{PiB}$ cortical retention ratios were higher than florbetapir values for $A \beta$-positive subjects, another explanation is that florbetapir has less gray 
matter plaque retention than $\mathrm{PiB}$ relative to a similar amount of white matter retention. These explanations, separately or together, may account for the higher $y$-intercept values in the $\mathrm{PiB}-$ florbetapir regression equations relative to the PiB1PiB2 equations (Fig. 3). The influence of white matter retention on estimation of cortical retention ratios for both tracers may also be exacerbated by image smoothing, possibly accounting for the slightly reduced PiB-florbetapir association with smoothed (rather than unsmoothed) image data.

Our findings suggest that image analysis methods such as spatial normalization and precise definition of cortical regions of interest minimally influence the quantification of cortical retention estimates. This result was surprising, because the methods were considerably different. The PET-template method involved spatial normalization of images and use of functionally defined cortical regions that were not restricted to gray matter, whereas the Freesurfer method used gray matter-specific regions of interest in subjects' native space. In addition, somewhat different sets of cortical voxels were included in the average cortical retention ratios for the 2 methods; for example, the PET-template method used regions that resulted from a statistical (voxelwise) contrast, whereas the Freesurfer method used anatomically defined regions that were limited to gray matter voxels only. Finally, whether the data were at uniform voxel size or resolution, and which reference region was used to normalize cortical retention ratios, had little impact on the PiB--florbetapir association, although the reference region did influence the scale of retention values (with use of the pons-brain stem resulting in the narrowest range of cortical values).

We also observed that cutoffs for establishing positive and negative $A \beta$ status could be accurately transformed between radioligands and processing methods. The PiB threshold of 1.47 (14) (based on cerebellar gray matter normalization and Freesurfer analysis) could be converted to a florbetapir threshold of 1.13 (based on whole cerebellum normalization and PET-template analysis), a value that is close to an independently derived florbetapir threshold of 1.10 (10). Existing A $\beta$ PET thresholds thus appear to have a high level of internal consistency, despite originating from separate datasets and processing methods. However, the PiB threshold was derived from a receiver-operating-characteristic analysis that has limited validity because it included in the negative-standard-oftruth group a proportion of amyloid-positive cognitively normal subjects. The 1.10 florbetapir threshold was therefore advantageous in that it has been further validated in histopathology studies $(5,6)$. This threshold has also been applied in recent longitudinal studies showing that $A \beta$-positive status in cognitively normal and MCI subjects was associated with greater cognitive decline than $A \beta$-negative status $(2,21)$.

An important limitation of this study was the relatively small size of this convenience sample and the considerable time intervals between the 2 sets of scans. Participants may have experienced changes in amyloid plaque load during the 1.5-y interval between their $\mathrm{PiB}$ and florbetapir scans. Indeed, 5 of $32 \mathrm{MCI}$ subjects converted to AD between their
PiB2 and florbetapir sessions; however, there was no evidence for a different pattern of associations for these subjects.

These analyses are an initial step in addressing the need for standardization of A $\beta$ PET methodologies. PET image data are currently acquired using a combination of radioligands, scanner types, and analysis methods, which has raised questions about differences in the criteria for positive-negative status across radioligands and analysis pipelines. Here, we demonstrate that despite these acquisition differences, reliable numeric conversions can be made.

\section{CONCLUSION}

Among the factors we examined in this study (PiB vs. florbetapir, level of preprocessing, image analysis method, reference region), we found that the radioligand used during scanning and the reference region used during image analysis have a substantive impact on the numeric scale of cortical retention ratios, whereas image preprocessing factors (degree of uniformity in voxel size and resolution uniformity) and image analysis methods (whether spatial normalization to a template occurred) had a minimal impact. However, differences in the scale of cortical retention ratios that result from the use of different radioligands can be accounted for by estimating conversion factors using a study population scanned with both radioligands. Conversion of a $\mathrm{PiB}$ cutoff to florbetapir units and vice versa resulted in excellent agreement in this population between subjects categorized as amyloid-positive and -negative using the converted cutoffs.

\section{DISCLOSURE}

The costs of publication of this article were defrayed in part by the payment of page charges. Therefore, and solely to indicate this fact, this article is hereby marked "advertisement" in accordance with 18 USC section 1734. Data collection and sharing for this project was funded by the ADNI (National Institutes of Health grant U01 AG024904). This research was also supported by Avid Radiopharmaceuticals, Inc.; NIH grants P30AG010129, K01 AG030514, and U01 AG024904; and the Dana Foundation.

ADNI is funded by the National Institute on Aging, the National Institute of Biomedical Imaging and Bioengineering, and through generous contributions from the following: Abbott, Alzheimer's Association, Alzheimer's Drug Discovery Foundation, Amorfix Life Sciences Ltd., AstraZeneca, Bayer HealthCare, BioClinica, Inc., Biogen Idec Inc., Bristol-Myers Squibb Co., Eisai Inc., Elan Pharmaceuticals Inc., Eli Lilly and Company, F. Hoffmann-La Roche Ltd. and its affiliated company Genentech, Inc., GE Healthcare, Innogenetics, N.V., IXICO Ltd., Janssen Alzheimer Immunotherapy Research \& Development, LLC, Johnson \& Johnson Pharmaceutical Research \& Development LLC, Medpace, Inc., Merck \& Co., Inc., Meso Scale Diagnostics, LLC, Novartis Pharmaceuticals Corp., Pfizer Inc., Servier, Synarc Inc., and Takeda Pharmaceutical Co.. The Canadian Institutes of Health Research is providing funds to support ADNI clinical sites in Canada. Private sector contributions are facilitated by the Foundation 
for the National Institutes of Health (www.fnih.org). The grantee organization is the Northern California Institute for Research and Education, and the study is coordinated by the Alzheimer's Disease Cooperative Study at the University of California, San Diego. ADNI data are disseminated by the Laboratory for Neuro Imaging at the University of California, Los Angeles. This research was also supported by NIH grants P30 AG010129 and K01 AG030514.

Susan Landau is a consultant for Avid Radiopharmaceuticals, Inc., and has previously consulted for Janssen Alzheimer Immunotherapy. Christopher Breault, Abhinay D. Joshi, Michael Pontecorvo, and Mark A. Mintun are employees of Avid Radiopharmaceuticals, Inc. Chester A. Mathis has a financial interest in a technology license agreement for PiB between GE Healthcare and the University of Pittsburgh and has consulted for GE Healthcare and Janssen Alzheimer's Immunotherapy. William Jagust collaborates with Avid Radiopharmaceuticals, Inc., through participation in the ADNI, has consulted in the past for GE Healthcare, and is currently a consultant to Genentech, Elan/ Janssen Alzheimer Immunotherapy, Synarc, and TauRx.

Data used in preparation of this article were obtained from the ADNI database (adni.loni.ucla.edu). As such, the investigators within the ADNI contributed to the design and implementation of ADNI or provided data but did not participate in analysis or writing of this report. A complete listing of ADNI investigators can be found at http://adni.loni.ucla.edu/wpcontent/ uploads/how_to_apply/ADNI_Acknowledgment_List.pdf.

No other potential conflict of interest relevant to this article was reported.

\section{ACKNOWLEDGMENTS}

We thank Beth Mormino, Cindee Madison, and Suzanne Baker for their contributions to data analysis.

\section{REFERENCES}

1. Fleisher AS, Chen K, Liu X, et al. Using positron emission tomography and florbetapir F18 to image cortical amyloid in patients with mild cognitive impairment or dementia due to Alzheimer disease. Arch Neurol. 2011;68:1404-1411.
2. Landau SM, Mintun M, Joshi A, et al. Amyloid deposition, hypometabolism, and longitudinal cognitive decline. Ann Neurol. 2012;72:578-586.

3. Rowe CC, Ellis KA, Rimajova M, et al. Amyloid imaging results from the Australian Imaging, Biomarkers and Lifestyle (AIBL) study of aging. Neurobiol Aging. 2010;31:1275-1283.

4. Ikonomovic MD, Klunk WE, Abrahamson EE, et al. Post-mortem correlates of in vivo PiB-PET amyloid imaging in a typical case of Alzheimer's disease. Brain. 2008;131:1630-1645.

5. Clark CM, Schneider JA, Bedell BJ, et al. Use of florbetapir-PET for imaging beta-amyloid pathology. JAMA. 2011;305:275-283.

6. Clark CM, Pontecorvo MJ, Beach TG, et al. Cerebral PET with florbetapir compared with neuropathology at autopsy for detection of neuritic amyloid-beta plaques: a prospective cohort study. Lancet Neurol. 2012;11: 669-678.

7. Wolk DA, Zhang Z, Boudhar S, Clark CM, Pontecorvo MJ, Arnold SE. Amyloid imaging in Alzheimer's disease: comparison of florbetapir and Pittsburgh compound-B positron emission tomography. J Neurol Neurosurg Psychiatry. 2012;83:923-926.

8. ADNI. Image data. Available at: http://adni.loni.ucla.edu/about-data-samples/imagedata/. Accessed October 24, 2012.

9. Joshi A, Koeppe RA, Fessler JA. Reducing between scanner differences in multicenter PET studies. Neuroimage. 2009;46:154-159.

10. Joshi AD, Pontecorvo MJ, Clark CM, et al. Performance characteristics of amyloid PET with florbetapir F 18 in patients with Alzheimer's disease and cognitively normal subjects. J Nucl Med. 2012;53:378-384.

11. Friston KJ, Ashburner J, Frith CD, Poline J-B, Heather JD, Frackowiak RSJ. Spatial registration and normalization of images. Hum Brain Mapp. 1995;2: 65-189.

12. Mormino EC, Kluth JT, Madison CM, et al. Episodic memory loss is related to hippocampal-mediated beta-amyloid deposition in elderly subjects. Brain. 2009; 132:1310-1323.

13. adni.loni.ucla.edu/research/pet-analysis/.

14. Jagust WJ, Landau SM, Shaw LM, et al. Relationships between biomarkers in aging and dementia. Neurology. 2009;73:1193-1199.

15. Villemagne VL, Mulligan RS, Pejoska S, et al. Comparison of ${ }^{11} \mathrm{C}-\mathrm{PiB}$ and ${ }^{18} \mathrm{~F}-$ florbetaben for Abeta imaging in ageing and Alzheimer's disease. Eur J Nucl Med Mol Imaging. 2012;39:983-989.

16. Wong DF, Rosenberg PB, Zhou Y, et al. In vivo imaging of amyloid deposition in Alzheimer disease using the radioligand ${ }^{18} \mathrm{~F}-\mathrm{AV}-45$ (florbetapir [corrected] F 18). J Nucl Med. 2010;51:913-920.

17. Vandenberghe R, Van Laere K, Ivanoiu A, et al. ${ }^{18}$ F-flutemetamol amyloid imaging in Alzheimer disease and mild cognitive impairment: a phase 2 trial. Ann Neurol. 2010;68:319-329.

18. Rowe CC, Ackerman U, Browne W, et al. Imaging of amyloid beta in Alzheimer's disease with ${ }^{18} \mathrm{~F}-\mathrm{BAY} 94-9172$, a novel PET tracer: proof of mechanism. Lancet Neurol. 2008;7:129-135.

19. Cselényi Z, Jonhagen ME, Forsberg A, et al. Clinical validation of ${ }^{18} \mathrm{~F}$-AZD4694, an amyloid-beta-specific PET radioligand. J Nucl Med. 2012;53:415-424.

20. Klunk WE, Engler H, Nordberg A, et al. Imaging brain amyloid in Alzheimer's disease with Pittsburgh Compound-B. Ann Neurol. 2004;55:306-319.

21. Doraiswamy PM, Sperling RA, Coleman RE, et al. Amyloid-beta assessed by florbetapir F 18 PET and 18-month cognitive decline: a multicenter study. Neurology. 2012;79:1636-1644. 